Journal of Bangladesh Academy of Sciences, Vol. 34, No. 1, 71-82, 2010

\title{
APPROXIMATE SOLUTION OF A FOURTH ORDER WEAKLY NON-LINEAR DIFFERENTIAL SYSTEM WITH STRONG DAMPING AND SLOWLY VARYING COEFFICIENTS BY UNIFIED KBM METHOD
}

\author{
M. ALHAZ UDDIN* AND M. A. SATTAR \\ Department of Mathematics, Rajshahi University, Rajshahi-6205, Bangladesh
}

\begin{abstract}
The unified Krylov-Bogoliubov-Mitropolskii (KBM) method is used for determining the analytical approximate solution of a fourth order weakly nonlinear differential system with strong damping and slowly varying coefficients when a pair of eigen-values of the unperturbed equation is a multiple (approximately or perfectly) of the other pair or pairs. In a damped case, one of the natural frequencies of the linearized equation may be a multiple of the other. The analytical first order approximate solution for different initial conditions shows a good coincidence with those obtained by the numerical procedure. The method is illustrated by an example.
\end{abstract}

Key words: Perturbation method, Weak nonlinearity, Oscillatory process, Strong damping, Varying coefficients

\section{INTRODUCTION}

The method of KBM is convenient and one of the widely used techniques to obtain the analytical approximate solutions of nonlinear differential systems. It is perhaps noteworthy that because of importance of physical process involving damping, Popov (1956) extended this method to damped oscillatory systems. Murty et al. (1971) used Popov's method to obtain over-damped solutions of nonlinear differential equations, which were the basis of unified theory of Murty (1969). Later this method has been extended to damped oscillatory and purely non oscillatory systems with slowly varying coefficients by Bojadziev and Edwards (1981). Arya and Bojadziev (1981) have studied a time-dependent nonlinear oscillatory system with damping, slowly varying coefficients and delay. Feshchenko et al. (1966) have presented a brief way to determine KBM (Krylov et al.1947, Bogoliubov et al. 1961)) solution (first order) of a second or third order nonlinear differential system. Arya and Bojadziev (1980) have also studied a system of second order nonlinear hyperbolic partial differential equation with slowly varying coefficients. Alam (1997) has investigated a unified KBM method for solving nonlinear system of order $n \geq 2$. Further, Alam (2002) has investigated a unified KBM method for solving of second and third nonlinear systems with constant coefficients. In another paper, Alam (2003) has also investigated a unified KBM method for solving nonlinear system of order $n \geq 3$ with slowly varying coefficients. Recently Alam and

\footnotetext{
*Author for correspondence. <alhazuddin@yahoo.com>.
} 
Sattar (2004) have also presented an asymptotic method for third order nonlinear system with slowly varying coefficients. Recently Akbar et al. (2006) have studied a fourth order nonlinear differential equation with constant coefficients. Most of the authors have studied the second and third order nonlinear differential systems for both constant and varying coefficients to obtain the analytical first order approximate solutions. The complicated and no less important case of a fourth order nonlinear differential equation with strong damping and slowly varying coefficients has remained almost untouched. The main goal of this paper is to fill this gap.

\section{METHOD}

Let us consider a fourth order weakly nonlinear ordinary differential equation with slowly varying coefficients in the following form:

$$
x^{(4)}+c_{1}(\tau) \dddot{x}+c_{2}(\tau) \ddot{x}+c_{3}(\tau) \dot{x}+c_{4}(\tau) x=\varepsilon f(x, \dot{x}, \ddot{x}, \dddot{x}, \tau)
$$

where the over dots represent the time derivatives, $\varepsilon$ is a small positive parameter which measures the strength of the nonlinearity, $\tau=\varepsilon t$ is the slowly varying time, $c_{j}(\tau) \geq 0$, $j=1,2,3,4$ are slowly varying coefficients and $f$ is a given nonlinear function. The coefficients are slowly varying in the sense that their time derivatives are proportional to $\varepsilon$ (Alam 2003).

By setting $\varepsilon=0, \tau=\tau_{0}=$ constant in Eq. (1), we obtain the solution of the unperturbed equation. We assume that the unperturbed equation of Eq. (1) has four eigenvalues $\lambda_{j}\left(\tau_{0}\right), j=1,2,3,4$; where $\lambda_{\mathrm{j}}\left(\tau_{0}\right)$ are constants, but if $\varepsilon \neq 0$ then $\lambda_{\mathrm{j}}(\tau)$ are varying slowly with time $t$. The solution of the linearized equation of Eq. (1) has the following form:

$$
x(t, 0)=\sum_{j=1}^{4} a_{j, 0} e^{\lambda_{j}\left(\tau_{0}\right) t}
$$

where $a_{j, 0}, j=1,2,3,4$ are arbitrary constants.

Now we are going to choose a solution of Eq. (1) that reduces to Eq. (2) as a limit $\varepsilon \rightarrow 0$ in the following form according to the KBM (Krylov et al. 1947, Bogoliubov et al. 1961) method:

$x(t, \varepsilon)=\sum_{j=1}^{4} a_{j}(t)+\varepsilon u_{1}\left(a_{1}, a_{2}, a_{3}, a_{4}, \tau\right)+\varepsilon^{2} u_{2}\left(a_{1}, a_{2}, a_{3}, a_{4}, \tau\right)+\varepsilon^{3} \cdots$,

where $u_{1}$ is a function of $a_{j}, j=1,2,3,4$ and each $a_{j}$ satisfies the following first order differential equation:

$\dot{a}_{j}=\lambda_{j} a_{j}+\varepsilon A_{j}\left(a_{1}, a_{2}, a_{3}, a_{4}, \tau\right)+\varepsilon^{2} B_{j}\left(a_{1}, a_{2}, a_{3}, a_{4}, \tau\right)+\varepsilon^{3} \cdots$ 
Confining only to the first few terms, 1, 2, 3... in the series expansions of Eq. (3) and Eq. (4), we evaluate the functions $u_{1}, u_{2}, \cdots$ and $A_{j}, B_{j}, \cdots, j=1,2,3,4$ such that each $a_{j}(t)$ appearing in Eq. (3) and Eq. (4) satisfies the given differential equation (1) with an accuracy of $\varepsilon^{m+1}$ (Alam 2003). In order to determine these functions it is assumed that the functions $u_{1}, u_{2}, \cdots$ do not contain the fundamental terms (Alam et al. 1997, Alam 2003, Bojadziev et al. 1981, Murty 1971) which are included in the series expansions (3) at order $\varepsilon^{0}$. Now differentiating Eq. (3) four-times with respect to time $t$ and using the relations Eq. (4) and by substituting the values of $x^{(4)}, \dddot{x}, \ddot{x}, \dot{x}$ and $x$ into the original Eq. (1) with the slowly varying coefficients

$$
\begin{gathered}
c_{1}(\tau)=-\left(\lambda_{1}(\tau)+\lambda_{2}(\tau)+\lambda_{3}(\tau)+\lambda_{4}(\tau)\right), \\
c_{2}(\tau)=\lambda_{1}(\tau) \lambda_{2}(\tau)+\lambda_{1}(\tau) \lambda_{3}(\tau)+\lambda_{1}(\tau) \lambda_{4}(\tau)+\lambda_{2}(\tau) \lambda_{3}(\tau)+\lambda_{2}(\tau) \lambda_{4}(\tau)+\lambda_{3}(\tau) \lambda_{4}(\tau), \\
\mathrm{c}_{3}(\tau)=-\left(\lambda_{1}(\tau) \lambda_{2}(\tau) \lambda_{3}(\tau)+\lambda_{1}(\tau) \lambda_{2}(\tau) \lambda_{4}(\tau)+\lambda_{1}(\tau) \lambda_{3}(\tau) \lambda_{4}(\tau)+\lambda_{2}(\tau) \lambda_{3}(\tau) \lambda_{4}(\tau)\right)
\end{gathered}
$$

and $\mathrm{C}_{4}(\tau)=\lambda_{1}(\tau) \lambda_{2}(\tau) \lambda_{3}(\tau) \lambda_{4}(\tau)$ and by expanding the right hand side of Eq. (1) by Taylor series and by equating the coefficients of $\varepsilon$ on both sides, we obtain the following equation:

$$
\begin{aligned}
& \prod_{j=1}^{4}\left(\Omega-\lambda_{j}\right) u_{1}+\sum_{j=1}^{4}\left(\prod_{k=1, k \neq j}^{4}\left(\Omega-\lambda_{k}\right) A_{j}\right)+\sum_{j=1}^{4} \frac{1}{2}\left(\sum_{k=0}^{2}(4-k)(3-k) c_{k} \lambda_{j}^{(2-k)}\right) \lambda_{j}^{\prime} a_{j} \\
& =f^{(0)}\left(a_{1}, a_{2}, a_{3}, a_{4}, \tau\right)
\end{aligned}
$$

where

$\Omega=\sum_{j=1}^{4} \lambda_{j} a_{j} \frac{\partial}{\partial a_{j}}, \lambda_{j}^{\prime}=\frac{d \lambda_{j}}{d \tau}, j=1.2, \cdots 4, f^{(0)}\left(a_{1}, a_{2}, a_{3}, a_{4}, \tau\right)=f\left(x_{0}, \dot{x}_{0}, \ddot{x}_{0}, \dddot{x}_{0}, \tau\right)$

and $\quad x_{0}=\sum_{j=1}^{4} a_{j}$

We have already assumed that $u_{1}$ does not contain the fundamental terms and for this reason the solution will be free from secular terms, namely $t \cos t, t \sin t$ and $t e^{-t}$. Under these restrictions, we are able to solve Eq. (5) by separating this into five individual equations for the unknown functions $u_{1}$ and $A_{j}$. In general, the functions $f^{(0)}$ and $u_{1}$ are expanded in Taylor's series in the following forms:

$$
\begin{aligned}
& f^{(0)}=\sum_{m_{1}=0, m_{2}=0, m_{3}=0 m_{4}=0}^{\infty, \infty, \infty, \infty} F_{m_{1}, m_{2}, m_{3}, m_{4}}(\tau) a_{1}^{m_{1}} a_{2}^{m_{2}} a_{3}^{m_{3}} a_{4}^{m_{4}}, \\
& u_{1}=\sum_{m_{1}=0, m_{2}=0, m_{3}=0 m_{4}=0}^{\infty, \infty, \infty, \infty} U_{m_{1}, m_{2}, m_{3}, m_{4}}(\tau) a_{1}^{m_{1}} a_{2}^{m_{2}} a_{3}^{m_{3}} a_{4}^{m_{4}} .
\end{aligned}
$$

The eigen-values of the unperturbed equation can be written as $-\mu_{l}\left(\tau_{0}\right) \pm i \omega_{l}\left(\tau_{0}\right)$, where $l=1,2$. For the above restrictions, it guaranties that $u_{1}$ must exclude all terms 
with $a_{2 l-1}^{m_{2 l-1}} a_{2 l}^{m_{2 l}}$ of $f^{(0)}$, where $m_{2 l-1}-m_{2 l}= \pm 1$. Since according to the linear approximation (i.e. $\varepsilon \rightarrow 0$ ), $a_{2 l-1}{ }^{m_{2 l-1}} a_{2 l}{ }^{m_{2 l}}$ becomes $e^{\omega_{l} t}$ when $m_{2 l-1}-m_{2 l}=1$ or $e^{-\omega_{l} t}$ when $m_{2 l-1}-m_{2 l}=-1$. It is noticed that $e^{ \pm \omega_{1} t}$ are known as the fundamental terms (Alam et al. 1997, Alam 2003, Bojdziev et al. 1981, Murty 1971). Usually these are included in equations $A_{j}$. Also, it is restricted (by Krylov et al. 1947, Bogoliubov et al. 1961) that the functions $A_{j}$ are independent of the fundamental terms.

Then the equations for $u_{1}$ and $A_{j}, j=1,2,3,4$ are written as

$$
\prod_{j=1}^{4}\left(\Omega-\lambda_{j}\right) u_{1}=\sum_{m_{2 l-1}=0, m_{2 l}=0}^{\infty, \infty} F_{, m_{L-1}, m_{2 l}}(\tau) a_{2 l-1}^{m_{l-1}} a_{2 l}^{m_{2 l}} \quad m_{2 l-1}-m_{2 l} \neq 0, \pm 1
$$

and

$$
\begin{gathered}
\left(\prod_{k=1, k \neq 2 l-1}^{4}\left(\Omega-\lambda_{k}\right)\right) A_{2 l-1}+\frac{1}{2}\left(\sum_{k=0}^{2}(4-k)(3-k) c_{k} \lambda_{2 l-1}^{(2 l-k-2)}\right) \lambda_{2 l-1}^{\prime} a_{2 l-1} \\
=\sum_{m_{2 l-1}=0, m_{2 l}=0}^{\infty, \infty} F_{m_{2 l}, m_{2 l}} a_{2 l-1}^{m_{2 l-1}} a_{2 l}^{m_{2 l}}, \quad m_{2 l-1}-m_{2 l}=1 \\
\left(\prod_{k=1, k \neq 2 l}^{4}\left(\Omega-\lambda_{k}\right)\right) A_{2 l}+\frac{1}{2}\left(\sum_{k=0}^{2}(4-k)(3-k) c_{k} \lambda_{2 l}^{(2 l-k-2)}\right) \lambda_{2 l}^{\prime} a_{2 l} \\
=\sum_{m_{2 l-1}=0, m_{2 l}=0}^{\infty, \infty} F_{m_{l l}, m_{2 l}} a_{2 l-1}^{m_{2 l-1}} a_{2 l}^{m_{2 l}}, \quad m_{2 l-1}-m_{2 l}=-1 .
\end{gathered}
$$

To determine the particular solutions of Eqs. (8) - (10), we have replaced the operator $\Omega$ by $\sum_{j=1}^{4} m_{j} \lambda_{j}$, since we know that $\Omega\left(a_{2 l-1}^{m_{l-1}} a_{2 l}^{m_{2 l}}\right)=\sum_{j=1}^{n} m_{j} \lambda_{j}\left(a_{2 l-1}^{m_{l-1}} a_{2 l}^{m_{2 l}}\right)$. Hence the determination of the analytical first order approximate solution of Eq. (1) is clear to us. We notice that the solution Eq. (3) is not a standard form of KBM method and is presented in terms of some unusual variables. Therefore, the solution obtained by formula of Eq. (1) is transformed to the formal form by replacing the unusual variables by amplitudes and phases variables in the forms:

$$
a_{2 l-1}=\frac{1}{2} b_{l} e^{i \varphi_{1}}, \quad a_{2 l}= \pm \frac{1}{2} b_{l} e^{-i \varphi_{l}}, l=1,2
$$

Thus the determination of the first order approximate solution of Eq. (1) is clear. The method can be carried out to higher order approximations in a similar way. However, owing to the rapidly growing algebraic complexity for the derivation of the formulae, the solution is in general confined to a low order, usually the first order (Krylov et al. 1947, Bogoliubov et al. 1961, Alam 2003, Akbar et al. 2006). 


\section{EXAMPLE}

To obtain the practical working of the above method, we consider the following fourth order weakly nonlinear differential equation with slowly varying coefficients in the following form:

$$
x^{(4)}+c_{1}(\tau) \dddot{x}+c_{2}(\tau) \ddot{x}+c_{3}(\tau) \dot{x}+c_{4}(\tau) x=\varepsilon x^{3},
$$

where $f(x, \dot{x}, \ddot{x}, \dddot{x}, \tau)=x^{3}$ and $x_{0}=a_{1}+a_{2}+a_{3}+a_{4}$.

Now

$$
\begin{aligned}
& f^{(0)}=a_{1}^{3}+a_{2}^{3}+a_{3}^{3}+a_{4}^{3}+3\left(a_{1}^{2} a_{2}+a_{1} a_{2}^{2}+a_{1}^{2} a_{3}+2 a_{1} a_{2} a_{3}+a_{2}^{2} a_{3}+a_{1}^{2} a_{4}\right. \\
& \left.2 a_{1} a_{2} a_{4} a_{2}^{2} a_{4}+a_{1} a_{3}^{2}+2 a_{1} a_{3} a_{4}+a_{1} a_{4}^{2}+a_{2} a_{3}^{2}+2 a_{2} a_{3} a_{4}+a_{2} a_{4}^{2}+a_{3}^{2} a_{4}+a_{3} a_{4}^{2}\right) .
\end{aligned}
$$

Substituting the values of $f^{(0)}$ in Eq. (5) and according to the above restrictions, we obtain five equations for $A_{1}, A_{2}, A_{3}, A_{4}$ and $u_{1}$ whose solutions are respectively given by

$$
\begin{aligned}
& A_{1}=-\frac{\left(3 \lambda_{1}^{2}-2 \lambda_{1} \lambda_{2}-2 \lambda_{1} \lambda_{3}-2 \lambda_{1} \lambda_{4}+\lambda_{2} \lambda_{3}+\lambda_{2} \lambda_{4}+\lambda_{3} \lambda_{4}\right) \lambda_{1}^{\prime} a_{1}}{\left(\lambda_{1}-\lambda_{2}\right)\left(\lambda_{1}-\lambda_{3}\right)\left(\lambda_{1}-\lambda_{4}\right)} \\
& +\frac{3 a_{1}^{2} a_{2}}{2 \lambda_{1}\left(2 \lambda_{1}+\lambda_{2}-\lambda_{3}\right)\left(2 \lambda_{1}+\lambda_{2}-\lambda_{4}\right)}+\frac{3 a_{1} a_{3}^{2}}{\left(\lambda_{1}+\lambda_{3}\right)\left(\lambda_{1}+2 \lambda_{3}-\lambda_{2}\right)\left(\lambda_{1}+2 \lambda_{3}-\lambda_{4}\right)} \\
& +\frac{3 a_{1} a_{4}^{2}}{\left(\lambda_{1}+\lambda_{4}\right)\left(\lambda_{1}+2 \lambda_{4}-\lambda_{2}\right)\left(\lambda_{1}+2 \lambda_{4}-\lambda_{3}\right)}+\frac{6 a_{1} a_{3} a_{4}}{\left(\lambda_{1}+\lambda_{3}\right)\left(\lambda_{1}+\lambda_{4}\right)\left(\lambda_{1}+\lambda_{3}+\lambda_{4}-\lambda_{2}\right)}, \\
& A_{2}=-\frac{\left(3 \lambda_{2}^{2}-2 \lambda_{1} \lambda_{2}-2 \lambda_{2} \lambda_{3}-2 \lambda_{2} \lambda_{4}+\lambda_{1} \lambda_{3}+\lambda_{1} \lambda_{4}+\lambda_{3} \lambda_{4}\right) \lambda_{2}^{\prime} a_{2}}{\left(\lambda_{2}-\lambda_{1}\right)\left(\lambda_{2}-\lambda_{3}\right)\left(\lambda_{2}-\lambda_{4}\right)} \\
& +\frac{3 a_{1} a_{2}^{2}}{2 \lambda_{2}\left(\lambda_{1}+2 \lambda_{2}-\lambda_{3}\right)\left(\lambda_{1}+2 \lambda_{2}-\lambda_{4}\right)}+\frac{3 a_{2} a_{3}^{2}}{\left(\lambda_{2}+\lambda_{3}\right)\left(\lambda_{2}+2 \lambda_{3}-\lambda_{1}\right)\left(\lambda_{2}+2 \lambda_{3}-\lambda_{4}\right)} \\
& +\frac{3 a_{2} a_{4}^{2}}{\left(\lambda_{2}+\lambda_{4}\right)\left(\lambda_{2}+2 \lambda_{4}-\lambda_{1}\right)\left(\lambda_{2}+2 \lambda_{4}-\lambda_{3}\right)}+\frac{6 a_{2} a_{3} a_{4}}{\left(\lambda_{2}+\lambda_{3}\right)\left(\lambda_{2}+\lambda_{4}\right)\left(\lambda_{2}+\lambda_{3}+\lambda_{4}-\lambda_{1}\right)}, \\
& A_{3}=-\frac{\left(3 \lambda_{3}^{2}-2 \lambda_{1} \lambda_{3}-2 \lambda_{2} \lambda_{3}-2 \lambda_{3} \lambda_{4}+\lambda_{1} \lambda_{2}+\lambda_{1} \lambda_{4}+\lambda_{2} \lambda_{4}\right) \lambda_{3}^{\prime} a_{3}}{\left(\lambda_{3}-\lambda_{1}\right)\left(\lambda_{3}-\lambda_{2}\right)\left(\lambda_{3}-\lambda_{4}\right)}+ \\
& \frac{3 a_{1}^{2} a_{3}}{\left(\lambda_{1}+\lambda_{3}\right)\left(2 \lambda_{1}+\lambda_{3}-\lambda_{2}\right)\left(2 \lambda_{1}+\lambda_{3}-\lambda_{4}\right)}+\frac{3 a_{2}^{2} a_{3}}{\left(\lambda_{2}+\lambda_{3}\right)\left(2 \lambda_{2}+\lambda_{3}-\lambda_{1}\right)\left(2 \lambda_{2}+\lambda_{3}-\lambda_{4}\right)} \\
& +\frac{3 a_{3}^{2} a_{4}}{2 \lambda_{3}\left(2 \lambda_{3}+\lambda_{4}-\lambda_{1}\right)\left(2 \lambda_{3}+\lambda_{4}-\lambda_{2}\right)}+\frac{6 a_{1} a_{3} a_{4}}{\left(\lambda_{1}+\lambda_{3}\right)\left(\lambda_{2}+\lambda_{3}\right)\left(\lambda_{1}+\lambda_{2}+\lambda_{3}-\lambda_{4}\right)}, \\
& A_{4}=-\frac{\left(3 \lambda_{4}^{2}-2 \lambda_{1} \lambda_{4}-2 \lambda_{2} \lambda_{4}-2 \lambda_{3} \lambda_{4}+\lambda_{1} \lambda_{2}+\lambda_{1} \lambda_{3}+\lambda_{2} \lambda_{3}\right) \lambda_{4}^{\prime} a_{4}}{\left(\lambda_{4}-\lambda_{1}\right)\left(\lambda_{4}-\lambda_{2}\right)\left(\lambda_{4}-\lambda_{3}\right)}+ \\
& \frac{3 a_{1}^{2} a_{4}}{\left(\lambda_{1}+\lambda_{4}\right)\left(2 \lambda_{1}+\lambda_{4}-\lambda_{2}\right)\left(2 \lambda_{1}+\lambda_{4}-\lambda_{3}\right)}+\frac{3 a_{2}^{2} a_{4}}{\left(\lambda_{2}+\lambda_{4}\right)\left(2 \lambda_{2}+\lambda_{4}-\lambda_{1}\right)\left(2 \lambda_{2}+\lambda_{4}-\lambda_{3}\right)} \\
& +\frac{3 a_{3} a_{4}^{2}}{2 \lambda_{4}\left(\lambda_{3}+2 \lambda_{4}-\lambda_{1}\right)\left(\lambda_{3}+2 \lambda_{4}-\lambda_{2}\right)}+\frac{6 a_{1} a_{2} a_{4}}{\left(\lambda_{1}+\lambda_{4}\right)\left(\lambda_{2}+\lambda_{4}\right)\left(\lambda_{1}+\lambda_{2}+\lambda_{4}-\lambda_{3}\right)},
\end{aligned}
$$


and

where

$$
u_{1}=C_{1} a_{1}^{3}+D_{1} a_{2}^{3}+C_{2} a_{3}^{3}+D_{2} a_{4}^{3},
$$

$$
\begin{aligned}
& C_{1}=\frac{1}{2 \lambda_{1}\left(3 \lambda_{1}-\lambda_{2}\right)\left(3 \lambda_{1}-\lambda_{3}\right)\left(3 \lambda_{1}-\lambda_{4}\right)}, D_{1}=\frac{1}{2 \lambda_{2}\left(3 \lambda_{2}-\lambda_{1}\right)\left(3 \lambda_{2}-\lambda_{3}\right)\left(3 \lambda_{2}-\lambda_{3}\right)}, \\
& C_{2}=\frac{1}{2 \lambda_{3}\left(3 \lambda_{3}-\lambda_{1}\right)\left(3 \lambda_{3}-\lambda_{2}\right)\left(3 \lambda_{3}-\lambda_{4}\right)}, D_{2}=\frac{1}{2 \lambda_{4}\left(3 \lambda_{4}-\lambda_{1}\right)\left(3 \lambda_{4}-\lambda_{2}\right)\left(3 \lambda_{4}-\lambda_{3}\right)} .
\end{aligned}
$$

Now by substituting the values of $A_{1}, A_{2}, A_{3}$ and $A_{4}$ from Eq. (14) into Eq. (4), we get

$$
\begin{aligned}
& \dot{a}_{1}=\lambda_{1} a_{1}+\varepsilon\left(-\frac{\left(3 \lambda_{1}^{2}-2 \lambda_{1} \lambda_{2}-2 \lambda_{1} \lambda_{3}-2 \lambda_{1} \lambda_{4}+\lambda_{2} \lambda_{3}+\lambda_{2} \lambda_{4}+\lambda_{3} \lambda_{4}\right) \lambda_{1}^{\prime} a_{1}}{\left(\lambda_{1}-\lambda_{2}\right)\left(\lambda_{1}-\lambda_{3}\right)\left(\lambda_{1}-\lambda_{4}\right)}\right. \\
& +\frac{3 a_{1}^{2} a_{2}}{2 \lambda_{1}\left(2 \lambda_{1}+\lambda_{2}-\lambda_{3}\right)\left(2 \lambda_{1}+\lambda_{2}-\lambda_{4}\right)}+\frac{3 a_{1} a_{3}^{2}}{\left(\lambda_{1}+\lambda_{3}\right)\left(\lambda_{1}+2 \lambda_{3}-\lambda_{2}\right)\left(\lambda_{1}+2 \lambda_{3}-\lambda_{4}\right)} \\
& +\frac{3 a_{1} a_{4}^{2}}{\left(\lambda_{1}+\lambda_{4}\right)\left(\lambda_{1}+2 \lambda_{4}-\lambda_{2}\right)\left(\lambda_{1}+2 \lambda_{4}-\lambda_{3}\right)}+\frac{6 a_{1} a_{3} a_{4}}{\left(\lambda_{1}+\lambda_{3}\right)\left(\lambda_{1}+\lambda_{4}\right)\left(\lambda_{1}+\lambda_{3}+\lambda_{4}-\lambda_{2}\right)}, \\
& \dot{a}_{2}=\lambda_{2} a_{2}+\varepsilon\left(-\frac{\left(3 \lambda_{2}^{2}-2 \lambda_{1} \lambda_{2}-2 \lambda_{2} \lambda_{3}-2 \lambda_{2} \lambda_{4}+\lambda_{1} \lambda_{3}+\lambda_{1} \lambda_{4}+\lambda_{3} \lambda_{4}\right) \lambda_{2}^{\prime} a_{2}}{\left(\lambda_{2}-\lambda_{1}\right)\left(\lambda_{2}-\lambda_{3}\right)\left(\lambda_{2}-\lambda_{4}\right)}\right. \\
& +\frac{3 a_{1} a_{2}^{2}}{2 \lambda_{2}\left(\lambda_{1}+2 \lambda_{2}-\lambda_{3}\right)\left(\lambda_{1}+2 \lambda_{2}-\lambda_{4}\right)}+\frac{3 a_{2} a_{3}^{2}}{\left(\lambda_{2}+\lambda_{3}\right)\left(\lambda_{2}+2 \lambda_{3}-\lambda_{1}\right)\left(\lambda_{2}+2 \lambda_{3}-\lambda_{4}\right)} \\
& +\frac{3 a_{2} a_{4}^{2}}{\left(\lambda_{2}+\lambda_{4}\right)\left(\lambda_{2}+2 \lambda_{4}-\lambda_{1}\right)\left(\lambda_{2}+2 \lambda_{4}-\lambda_{3}\right)}+\frac{6 a_{2} a_{3} a_{4}}{\left(\lambda_{2}+\lambda_{3}\right)\left(\lambda_{2}+\lambda_{4}\right)\left(\lambda_{2}+\lambda_{3}+\lambda_{4}-\lambda_{1}\right)} \text {, } \\
& \dot{a}_{3}=\lambda_{3} a_{3}+\varepsilon\left(-\frac{\left(3 \lambda_{3}^{2}-2 \lambda_{1} \lambda_{3}-2 \lambda_{2} \lambda_{3}-2 \lambda_{3} \lambda_{4}+\lambda_{1} \lambda_{2}+\lambda_{1} \lambda_{4}+\lambda_{2} \lambda_{4}\right) \lambda_{3}^{\prime} a_{3}}{\left(\lambda_{3}-\lambda_{1}\right)\left(\lambda_{3}-\lambda_{2}\right)\left(\lambda_{3}-\lambda_{4}\right)}+\right. \\
& \frac{3 a_{1}^{2} a_{3}}{\left(\lambda_{1}+\lambda_{3}\right)\left(2 \lambda_{1}+\lambda_{3}-\lambda_{2}\right)\left(2 \lambda_{1}+\lambda_{3}-\lambda_{4}\right)}+\frac{3 a_{2}^{2} a_{3}}{\left(\lambda_{2}+\lambda_{3}\right)\left(2 \lambda_{2}+\lambda_{3}-\lambda_{1}\right)\left(2 \lambda_{2}+\lambda_{3}-\lambda_{4}\right)} \\
& +\frac{3 a_{3}^{2} a_{4}}{2 \lambda_{3}\left(2 \lambda_{3}+\lambda_{4}-\lambda_{1}\right)\left(2 \lambda_{3}+\lambda_{4}-\lambda_{2}\right)}+\frac{6 a_{1} a_{3} a_{4}}{\left(\lambda_{1}+\lambda_{3}\right)\left(\lambda_{2}+\lambda_{3}\right)\left(\lambda_{1}+\lambda_{2}+\lambda_{3}-\lambda_{4}\right)}, \\
& \dot{a}_{4}=\lambda_{4} a_{4}+\varepsilon\left(-\frac{\left(3 \lambda_{4}^{2}-2 \lambda_{1} \lambda_{4}-2 \lambda_{2} \lambda_{4}-2 \lambda_{3} \lambda_{4}+\lambda_{1} \lambda_{2}+\lambda_{1} \lambda_{3}+\lambda_{2} \lambda_{3}\right) \lambda_{4}^{\prime} a_{4}}{\left(\lambda_{4}-\lambda_{1}\right)\left(\lambda_{4}-\lambda_{2}\right)\left(\lambda_{4}-\lambda_{3}\right)}+\right. \\
& \frac{3 a_{1}^{2} a_{4}}{\left(\lambda_{1}+\lambda_{4}\right)\left(2 \lambda_{1}+\lambda_{4}-\lambda_{2}\right)\left(2 \lambda_{1}+\lambda_{4}-\lambda_{3}\right)}+\frac{3 a_{2}^{2} a_{4}}{\left(\lambda_{2}+\lambda_{4}\right)\left(2 \lambda_{2}+\lambda_{4}-\lambda_{1}\right)\left(2 \lambda_{2}+\lambda_{4}-\lambda_{3}\right)} \\
& +\frac{3 a_{3} a_{4}^{2}}{2 \lambda_{4}\left(\lambda_{3}+2 \lambda_{4}-\lambda_{1}\right)\left(\lambda_{3}+2 \lambda_{4}-\lambda_{2}\right)}+\frac{6 a_{1} a_{2} a_{4}}{\left(\lambda_{1}+\lambda_{4}\right)\left(\lambda_{2}+\lambda_{4}\right)\left(\lambda_{1}+\lambda_{2}+\lambda_{4}-\lambda_{3}\right)} .
\end{aligned}
$$


For a damped solution of Eq. (12), we may substitute $\lambda_{1,2}=-\mu_{1}(\tau) \pm \mathrm{i} \omega_{1}(\tau)$, $\lambda_{3,4}=-\mu_{2}(\tau) \pm i \omega_{2}(\tau)$ and using the transformation equations $a_{1}=\frac{1}{2} a e^{i \varphi_{1}}$, $a_{2}=\frac{1}{2} a e^{-i \varphi_{1}} a_{3}=\frac{1}{2} b e^{i \varphi_{2}}, a_{4}=\frac{1}{2} b e^{-i \varphi_{2}}$ into Eq. (17) and by separating the real and imaginary parts, we obtain the following variational equations for the amplitudes and phases variables:

$$
\begin{aligned}
& \dot{a}=-\mu_{1} a+\varepsilon\left(l_{1} a+l_{2} a^{3}+l_{3} a b^{2}+a b^{2}\left(E_{1} \cos 2 \varphi_{2}+F_{1} \sin 2 \varphi_{2}\right)\right), \\
& \dot{\varphi}_{1}=\omega_{1}(\tau)+\varepsilon\left(m_{1}+m_{2} a^{2}+m_{3} b^{2}+b^{2}\left(E_{2} \cos 2 \varphi_{2}+F_{2} \sin 2 \varphi_{2}\right)\right), \\
& \dot{b}=-\mu_{2} b+\varepsilon\left(p_{1} b+p_{2} a^{2} b+p_{3} b^{3}+a^{2} b\left(E_{3} \cos 2 \varphi_{1}+F_{3} \sin 2 \varphi_{1}\right)\right), \\
& \dot{\varphi}_{2}=\omega_{2}(\tau)+\varepsilon\left(q_{1}+q_{2} a^{2}+q_{3} b^{2}+a^{2}\left(E_{4} \cos 2 \varphi_{1}+F_{4} \sin 2 \varphi_{1}\right)\right),
\end{aligned}
$$

and the first correction term $u_{1}$ is obtained as

$$
u_{1}=a^{3}\left(c_{1} \cos 3 \varphi_{1}+d_{1} \sin 3 \varphi_{1}\right)+b^{3}\left(c_{2} \cos 3 \varphi_{2}+d_{2} \sin 3 \varphi_{2}\right) \text {, }
$$

where

$$
\begin{gathered}
\left(\omega_{1}^{\prime}\left(\left(\left(\mu_{1}-\mu_{2}\right)^{2}-5 \omega_{1}^{2}+\omega_{2}^{2}\right)\left(\left(\mu_{1}-\mu_{2}\right)^{2}-\omega_{1}^{2}+\omega_{2}^{2}\right)+12 \omega_{1}^{2}\left(\mu_{1}-\mu_{2}\right)^{2}\right)\right. \\
l_{1}=-\frac{\left.+4 \mu_{1}^{\prime} \omega_{1}\left(\mu_{1}-\mu_{2}\right)\left(\left(\mu_{1}-\mu_{2}\right)^{2}+\omega_{1}^{2}+\omega_{2}^{2}\right)\right)}{2 \omega_{1}\left(\left(\mu_{1}-\mu_{2}\right)^{2}+\left(\omega_{1}-\omega_{2}\right)^{2}\right)\left(\left(\mu_{1}-\mu_{2}\right)^{2}+\left(\omega_{1}+\omega_{2}\right)^{2}\right)} \\
m_{1}=\frac{\left.\left(\left(\mu_{1}-\mu_{2}\right)^{2}-\omega_{1}^{2}+\omega_{2}^{2}\right)+12 \omega_{1}^{2}\left(\mu_{1}-\mu_{2}\right)^{2}\right)}{2 \omega_{1}\left(\left(\mu_{1}-\mu_{2}\right)^{2}+\left(\omega_{1}-\omega_{2}\right)^{2}\right)\left(\left(\mu_{1}-\mu_{2}\right)^{2}+\left(\omega_{1}+\omega_{2}\right)^{2}\right)}, \\
l_{2}=-\frac{3\left(\mu_{1}\left(\left(3 \mu_{1}-\mu_{2}\right)^{2}-\omega_{1}^{2}+\omega_{2}^{2}\right)-2 \omega_{1}^{2}\left(3 \mu_{1}-\mu_{2}\right)\right)}{8\left(\mu_{1}^{2}+\omega_{1}^{2}\right)\left(\left(3 \mu_{1}-\mu_{2}\right)^{2}+\left(\omega_{1}-\omega_{2}\right)^{2}\right)\left(\left(3 \mu_{1}-\mu_{2}\right)^{2}+\left(\omega_{1}+\omega_{2}\right)^{2}\right)}, \\
m_{2}=-\frac{3 \omega_{1}\left(\left(3 \mu_{1}-\mu_{2}\right)\left(5 \mu_{1}-\mu_{2}\right)-\omega_{1}^{2}+\omega_{2}^{2}\right)}{8\left(\mu_{1}^{2}+\omega_{1}^{2}\right)\left(\left(3 \mu_{1}-\mu_{2}\right)^{2}+\left(\omega_{1}-\omega_{2}\right)^{2}\right)\left(\left(3 \mu_{1}-\mu_{2}\right)^{2}+\left(\omega_{1}+\omega_{2}\right)^{2}\right)}, \\
l_{3}=-\frac{3\left(\mu_{2}\left(\left(\mu_{1}+\mu_{2}\right)^{2}-\omega_{1}^{2}+\omega_{2}^{2}\right)-2 \omega_{2}^{2}\left(\mu_{1}+\mu_{2}\right)\right)}{4\left(\mu_{2}^{2}+\omega_{1}^{2}\right)\left(\left(\mu_{1}+\mu_{2}\right)^{2}+\left(\omega_{1}-\omega_{2}\right)^{2}\right)\left(\left(\mu_{1}+\mu_{2}\right)^{2}+\left(\omega_{1}+\omega_{2}\right)^{2}\right)}, \\
m_{3}=-\frac{3\left(\omega_{2}\left(\left(\mu_{1}+\mu_{2}\right)\left(\mu_{1}+3 \mu_{2}\right)-\omega_{1}^{2}+\omega_{2}^{2}\right)\right.}{8\left(\mu_{2}^{2}+\left(\omega_{1}+\omega_{2}\right)^{2}\right)\left(\left(\mu_{1}+\mu_{2}\right)^{2}+\left(\omega_{1}+\omega_{2}\right)^{2}\right)\left(\left(\mu_{1}+\mu_{2}\right)^{2}+\left(\omega_{1}+3 \omega_{2}\right)^{2}\right)},
\end{gathered}
$$




$$
\begin{aligned}
& l_{4}^{*}=\frac{3\left(2 \mu_{2}\left(\mu_{1}+\mu_{2}\right)\left(\omega_{1}+2 \omega_{2}\right)+\left(\omega_{1}+\omega_{2}\right)\left(\left(\mu_{1}+\mu_{2}\right)^{2}-\left(\omega_{1}+\omega_{2}\right)\left(\omega_{1}+3 \omega_{2}\right)\right)\right)}{8\left(\mu_{2}^{2}+\left(\omega_{1}+\omega_{2}\right)^{2}\right)\left(\left(\mu_{1}+\mu_{2}\right)^{2}+\left(\omega_{1}+\omega_{2}\right)^{2}\right)\left(\left(\mu_{1}+\mu_{2}\right)^{2}+\left(\omega_{1}+3 \omega_{2}\right)^{2}\right)}, \\
& m_{4}=-l_{4}{ }^{*}, m_{4}{ }^{*}=l_{4} \text {, } \\
& l_{5}=-\frac{3\left(\mu_{2}\left(\left(\mu_{1}+\mu_{2}\right)^{2}-\left(\omega_{1}-\omega_{2}\right)\left(\omega_{1}-3 \omega_{2}\right)\right)-2\left(\mu_{1}+\mu_{2}\right)\left(\omega_{1}-\omega_{2}\right)\left(\omega_{1}-2 \omega_{2}\right)\right)}{8\left(\mu_{2}^{2}+\left(\omega_{1}-\omega_{2}\right)^{2}\right)\left(\left(\mu_{1}+\mu_{2}\right)^{2}+\left(\omega_{1}-\omega_{2}\right)^{2}\right)\left(\left(\mu_{1}+\mu_{2}\right)^{2}+\left(\omega_{1}-3 \omega_{2}\right)^{2}\right)}, \\
& l_{5}^{*}=-\frac{3\left(2 \mu_{2}\left(\mu_{1}+\mu_{2}\right)\left(\omega_{1}-2 \omega_{2}\right)+\left(\omega_{1}-\omega_{2}\right)\left(\left(\mu_{1}+\mu_{2}\right)^{2}-\left(\omega_{1}-\omega_{2}\right)\left(\omega_{1}-3 \omega_{2}\right)\right)\right)}{8\left(\mu_{2}^{2}+\left(\omega_{1}-\omega_{2}\right)^{2}\right)\left(\left(\mu_{1}+\mu_{2}\right)^{2}+\left(\omega_{1}-\omega_{2}\right)^{2}\right)\left(\left(\mu_{1}+\mu_{2}\right)^{2}+\left(\omega_{1}-3 \omega_{2}\right)^{2}\right)}, \\
& m_{5}=l_{5}^{*}, m_{4}^{*}=-l_{5} \text {, } \\
& \left(\omega_{2}^{\prime}\left(\left(\left(\mu_{1}-\mu_{2}\right)^{2}+\omega_{1}^{2}-\omega_{2}^{2}\right)\left(\left(\mu_{1}-\mu_{2}\right)^{2}+\omega_{1}^{2}-5 \omega_{2}^{2}\right)+12 \omega_{2}^{2}\left(\mu_{1}-\mu_{2}\right)^{2}\right)\right. \\
& p_{1}=-\frac{\left.-4 \mu_{2}^{\prime} \omega_{2}\left(\mu_{1}-\mu_{2}\right)\left(\left(\mu_{1}-\mu_{2}\right)^{2}+\omega_{1}^{2}+\omega_{2}^{2}\right)\right)}{2 \omega_{2}\left(\left(\mu_{1}-\mu_{2}\right)^{2}+\left(\omega_{1}-\omega_{2}\right)^{2}\right)\left(\left(\mu_{1}-\mu_{2}\right)^{2}+\left(\omega_{1}+\omega_{2}\right)^{2}\right)} \text {, } \\
& \left(4 \omega_{2} \omega_{2}^{\prime}\left(\mu_{1}-\mu_{2}\right)\left(\left(\mu_{1}-\mu_{2}\right)^{2}+\omega_{1}^{2}+\omega_{2}^{2}\right)+\mu_{2}^{\prime}\left(\left(\left(\mu_{1}-\mu_{2}\right)^{2}+\omega_{1}^{2}-\omega_{2}^{2}\right)\right.\right. \\
& q_{1}=-\frac{\left.\left(\left(\mu_{1}-\mu_{2}\right)^{2}+\omega_{1}^{2}-5 \omega_{2}^{2}\right)+12 \omega_{2}^{2}\left(\mu_{1}-\mu_{2}\right)^{2}\right)}{2 \omega_{2}\left(\left(\mu_{1}-\mu_{2}\right)^{2}+\left(\omega_{1}-\omega_{2}\right)^{2}\right)\left(\left(\mu_{1}-\mu_{2}\right)^{2}+\left(\omega_{1}+\omega_{2}\right)^{2}\right)} \text {, } \\
& p_{2}=-\frac{3\left(\mu_{1}\left(\left(\mu_{1}+\mu_{2}\right)^{2}+\omega_{1}^{2}-\omega_{2}^{2}\right)-2 \omega_{2}^{2}\left(\mu_{1}+\mu_{2}\right)\right)}{4\left(\mu_{1}^{2}+\omega_{2}^{2}\right)\left(\left(\mu_{1}+\mu_{2}\right)^{2}+\left(\omega_{1}+\omega_{2}\right)^{2}\right)\left(\left(\mu_{1}+\mu_{2}\right)^{2}+\left(\omega_{1}-\omega_{2}\right)^{2}\right)}, \\
& q_{2}=-\frac{3 \omega_{2}\left(\left(\mu_{1}+\mu_{2}\right)\left(3 \mu_{1}+\mu_{2}\right)+\omega_{1}^{2}-\omega_{2}^{2}\right)}{4\left(\mu_{1}^{2}+\omega_{2}^{2}\right)\left(\left(\mu_{1}+\mu_{2}\right)^{2}+\left(\omega_{1}+\omega_{2}\right)^{2}\right)\left(\left(\mu_{1}+\mu_{2}\right)^{2}+\left(\omega_{1}-\omega_{2}\right)^{2}\right)}, \\
& p_{3}=-\frac{3\left(\mu_{2}\left(\left(\mu_{1}-3 \mu_{2}\right)^{2}+\omega_{1}^{2}-\omega_{2}^{2}\right)+2 \omega_{2}^{2}\left(\mu_{1}-3 \mu_{2}\right)\right)}{8\left(\mu_{2}^{2}+\omega_{2}^{2}\right)\left(\left(\mu_{1}-3 \mu_{2}\right)^{2}+\left(\omega_{1}-\omega_{2}\right)^{2}\right)\left(\left(\mu_{1}-3 \mu_{2}\right)^{2}+\left(\omega_{1}+\omega_{2}\right)^{2}\right)}, \\
& q_{3}=-\frac{3 \omega_{2}\left(\left(\mu_{1}-3 \mu_{2}\right)\left(\mu_{1}-5 \mu_{2}\right)+\omega_{1}^{2}-\omega_{2}^{2}\right)}{8\left(\mu_{2}^{2}+\omega_{2}^{2}\right)\left(\left(\mu_{1}-3 \mu_{2}\right)^{2}+\left(\omega_{1}-\omega_{2}\right)^{2}\right)\left(\left(\mu_{1}-3 \mu_{2}\right)^{2}+\left(\omega_{1}+\omega_{2}\right)^{2}\right)}, \\
& p_{4}=-\frac{3\left(\mu_{1}\left(\left(\mu_{1}+\mu_{2}\right)^{2}-\left(\omega_{1}+\omega_{2}\right)\left(3 \omega_{1}+\omega_{2}\right)\right)-2\left(\mu_{1}+\mu_{2}\right)\left(\omega_{1}+\omega_{2}\right)\left(2 \omega_{1}+\omega_{2}\right)\right)}{8\left(\mu_{1}^{2}+\left(\omega_{1}+\omega_{2}\right)^{2}\right)\left(\left(\mu_{1}+\mu_{2}\right)^{2}+\left(\omega_{1}+\omega_{2}\right)^{2}\right)\left(\left(\mu_{1}+\mu_{2}\right)^{2}+\left(3 \omega_{1}+\omega_{2}\right)^{2}\right)}, \\
& p_{4}^{*}=\frac{3\left(2 \mu_{1}\left(\mu_{1}+\mu_{2}\right)\left(2 \omega_{1}+\omega_{2}\right)+\left(\omega_{1}+\omega_{2}\right)\left(\left(\mu_{1}+\mu_{2}\right)^{2}-\left(\omega_{1}+\omega_{2}\right)\left(3 \omega_{1}+\omega_{2}\right)\right)\right)}{8\left(\mu_{1}^{2}+\left(\omega_{1}+\omega_{2}\right)^{2}\right)\left(\left(\mu_{1}+\mu_{2}\right)^{2}+\left(\omega_{1}+\omega_{2}\right)^{2}\right)\left(\left(\mu_{1}+\mu_{2}\right)^{2}+\left(3 \omega_{1}+\omega_{2}\right)^{2}\right)}, \\
& q_{4}=-p_{4}^{*}, q_{4}^{*}=p_{4} \text {, }
\end{aligned}
$$




$$
\begin{aligned}
& p_{5}=-\frac{3\left(\mu_{1}\left(\left(\mu_{1}+\mu_{2}\right)^{2}-\left(\omega_{1}-\omega_{2}\right)\left(3 \omega_{1}-\omega_{2}\right)\right)-2\left(\mu_{1}+\mu_{2}\right)\left(\omega_{1}-\omega_{2}\right)\left(2 \omega_{1}-\omega_{2}\right)\right)}{8\left(\mu_{1}^{2}+\left(\omega_{1}-\omega_{2}\right)^{2}\right)\left(\left(\mu_{1}+\mu_{2}\right)^{2}+\left(\omega_{1}-\omega_{2}\right)^{2}\right)\left(\left(\mu_{1}+\mu_{2}\right)^{2}+\left(3 \omega_{1}-\omega_{2}\right)^{2}\right)}, \\
& p_{5}^{*}=\frac{3\left(2 \mu_{1}\left(\mu_{1}+\mu_{2}\right)\left(2 \omega_{1}-\omega_{2}\right)+\left(\omega_{1}-\omega_{2}\right)\left(\left(\mu_{1}+\mu_{2}\right)^{2}-\left(\omega_{1}-\omega_{2}\right)\left(3 \omega_{1}-\omega_{2}\right)\right)\right)}{8\left(\mu_{1}^{2}+\left(\omega_{1}-\omega_{2}\right)^{2}\right)\left(\left(\mu_{1}+\mu_{2}\right)^{2}+\left(\omega_{1}-\omega_{2}\right)^{2}\right)\left(\left(\mu_{1}+\mu_{2}\right)^{2}+\left(3 \omega_{1}-\omega_{2}\right)^{2}\right)}, \\
& q_{5}=p_{5}^{*}, q_{5}^{*}=-p_{5}, \\
& E_{1}=l_{4}+l_{5}, F_{1}=l_{4}^{*}+l_{5}^{*}, E_{2}=m_{4}+m_{5}, F_{2}=m_{4}^{*}+m_{5}^{*}, \\
& E_{3}=p_{4}+p_{5}, F_{3}=p_{4}{ }^{*}+p_{5}{ }^{*}, E_{4}=q_{4}+q_{5}, F_{4}=q_{4}{ }^{*}+q_{5}^{*},
\end{aligned}
$$

and

$$
\begin{aligned}
& c_{1}=\frac{\left(\mu_{1}^{2}-2 \omega_{1}^{2}\right)\left(\left(3 \mu_{1}-\mu_{2}\right)^{2}-9 \omega_{1}^{2}+\omega_{2}^{2}\right)-18 \mu_{1} \omega_{1}^{2}\left(3 \mu_{1}-\mu_{2}\right)}{16\left(\mu_{1}^{2}+\omega_{1}^{2}\right)\left(\mu_{1}^{2}+4 \omega_{1}^{2}\right)\left(\left(3 \mu_{1}-\mu_{2}\right)^{2}+\left(3 \omega_{1}-\omega_{2}\right)^{2}\right)\left(\left(3 \mu_{1}-\mu_{2}\right)^{2}+\left(3 \omega_{1}+\omega_{2}\right)^{2}\right)}, \\
& d_{1}=-\frac{3 \omega_{1}\left(\mu_{1}\left(\left(3 \mu_{1}-\mu_{2}\right)^{2}-9 \omega_{1}^{2}+\omega_{2}^{2}\right)+2\left(3 \mu_{1}-\mu_{2}\right)\left(\mu_{1}^{2}-2 \omega_{1}^{2}\right)\right)}{16\left(\mu_{1}^{2}+\omega_{1}^{2}\right)\left(\mu_{1}^{2}+4 \omega_{1}^{2}\right)\left(\left(3 \mu_{1}-\mu_{2}\right)^{2}+\left(3 \omega_{1}-\omega_{2}\right)^{2}\right)\left(\left(3 \mu_{1}-\mu_{2}\right)^{2}+\left(3 \omega_{1}+\omega_{2}\right)^{2}\right)}, \\
& c_{2}=\frac{\left(\mu_{2}^{2}-2 \omega_{2}^{2}\right)\left(\left(\mu_{1}-3 \mu_{2}\right)^{2}+\omega_{1}^{2}-9 \omega_{2}^{2}\right)+18 \mu_{2} \omega_{2}^{2}\left(\mu_{1}-3 \mu_{2}\right)}{16\left(\mu_{2}^{2}+\omega_{2}^{2}\right)\left(\mu_{2}^{2}+4 \omega_{2}^{2}\right)\left(\left(\mu_{1}-3 \mu_{2}\right)^{2}+\left(\omega_{1}-3 \omega_{2}\right)^{2}\right)\left(\left(\mu_{1}-3 \mu_{2}\right)^{2}+\left(\omega_{1}+3 \omega_{2}\right)^{2}\right)}, \\
& d_{2}=-\frac{3 \omega_{2}\left(\mu_{2}\left(\left(\mu_{1}-3 \mu_{2}\right)^{2}+\omega_{1}^{2}-9 \omega_{2}^{2}\right)-2\left(\mu_{1}-3 \mu_{2}\right)\left(\mu_{2}^{2}-2 \omega_{2}^{2}\right)\right)}{16\left(\mu_{2}^{2}+\omega_{2}^{2}\right)\left(\mu_{2}^{2}+4 \omega_{2}^{2}\right)\left(\left(\mu_{1}-3 \mu_{2}\right)^{2}+\left(\omega_{1}-3 \omega_{2}\right)^{2}\right)\left(\left(\mu_{1}-3 \mu_{2}\right)^{2}+\left(\omega_{1}+3 \omega_{2}\right)^{2}\right)} .
\end{aligned}
$$

Thus the analytical first order approximate solution (improved) of Eq. (12) is obtained by $x(t, \varepsilon)=a \cos \varphi_{1}+b \cos \varphi_{2}+\varepsilon u_{1}$,

where the amplitudes $a, b$ and phases $\varphi_{1}, \varphi_{2}$ are the solutions of Eq. (18) and $u_{1}$ is given by Eq. (19).

\section{RESULTS AND DISCUSSION}

A standard form of KBM method is presented to obtain the analytical approximate solution of a fourth order nonlinear differential equation with strong damping and slowly varying coefficients with small nonlinearity. The KBM method was originally developed for obtaining the periodic solutions of second-order nonlinear systems by Krylov and Bogoliubov (1947) and later it was amplified and justified by Bogoliubov and Mitropolskii (1961). The method is not only limited to second-order nonlinear problems, but also useful in third-order (Alam 2002, 2003) and fourth order (Akbar 2006) nonlinear systems. A general solution has been found for the damped nonlinear differential equation with slowly varying coefficients based on the unified KBM (Alam et al. 1997, Alam 2002, 2003, Bogoliubov et al. 1961, Krylov et al. 1947) method. 
We have solved four simultaneous differential equations for amplitude(s) and phase(s) variables and a partial differential equation for $u_{1}$ involving four independent variables of amplitudes and phases. Also we are able to solve all the equations of $A_{j}, j=1,2,3,4$ and $u_{1}$ by a unified formula. In a particular case, we are forced to assume that $\mu_{l}(\tau), l=1,2$ are constants, $\omega_{1}(\tau)=2 \omega_{2}(\tau)$ and $\omega_{2}(\tau)=\omega_{0} e^{-h \tau}$ are varying slowly with time $t$, where $\omega_{0}$ and $h$ are constants. Figures are drawn to compare between the analytical first order approximate solutions obtained by the perturbation method and those obtained by the numerical procedure (fourth-order Runge-Kutta method) for several damping effects. Moreover, this method is able to give the required results when the coefficients of the given nonlinear differential equation become constants $(h=0)$. From the Figs. (1)- (2), it is seen that the new analytical approximate solution shows a good agreement with the corresponding numerical solution (considered to be exact).

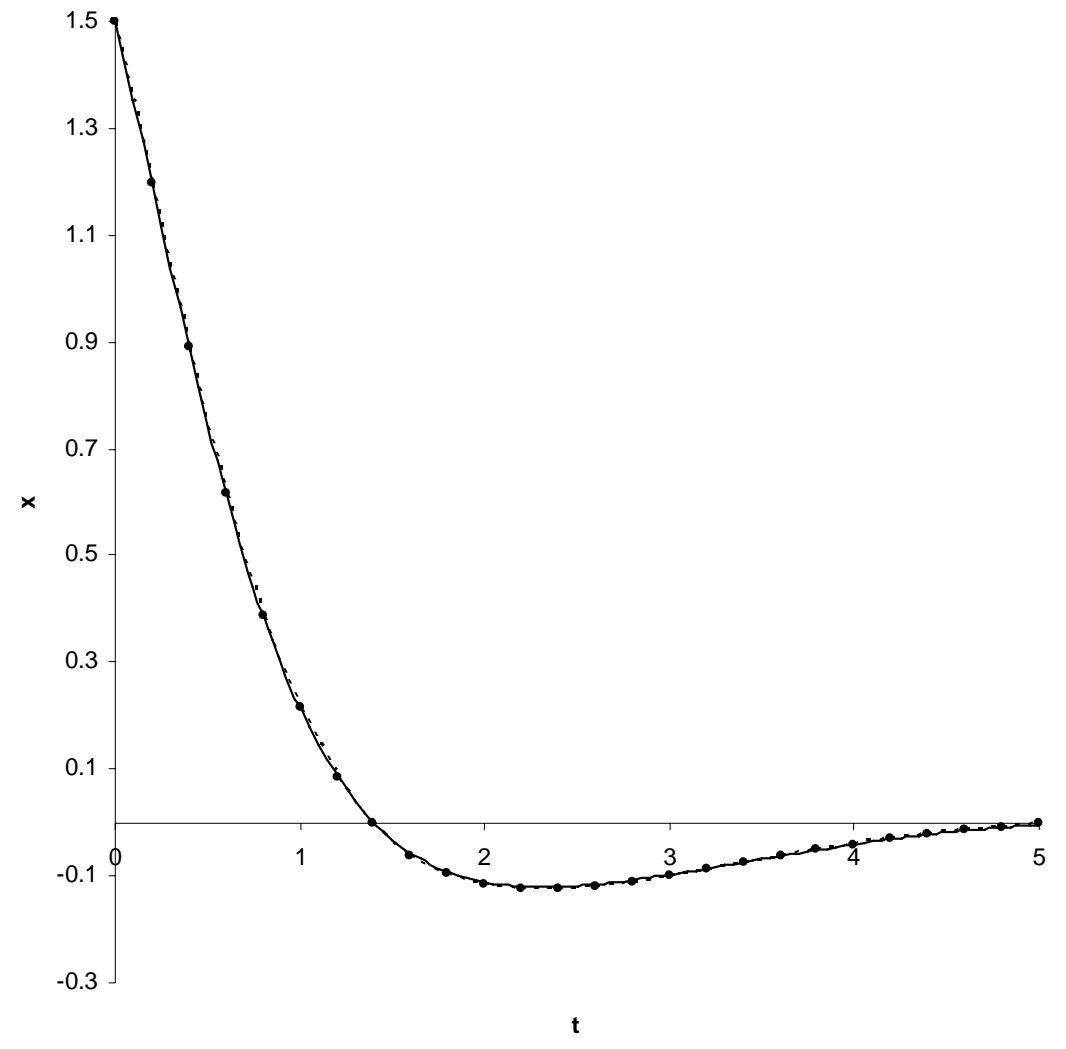

Fig. 1. First approximate solution (denoted by - - - ) of Eq. (13), with the initial conditions $[x(0)$ $=1.49992, \dot{x}(0)=-1.43242, \ddot{x}(0)=-1.43652, \dddot{x}(0)=8.767772]$ or $a_{0}=1.5, \varphi_{1}=0, b=$ $1.0 \varphi_{2}=0$, with $\mu_{1}=1.5, \mu_{2}=0.75, \omega_{0}=1.0, h=0.5, \varepsilon=0.1, \omega_{1}=2 \omega_{2}, \omega_{2}=\omega_{0} e^{-h r}, \tau=\varepsilon t$ and $f=x^{3}$. Corresponding numerical solution is denoted by - (solid line). 


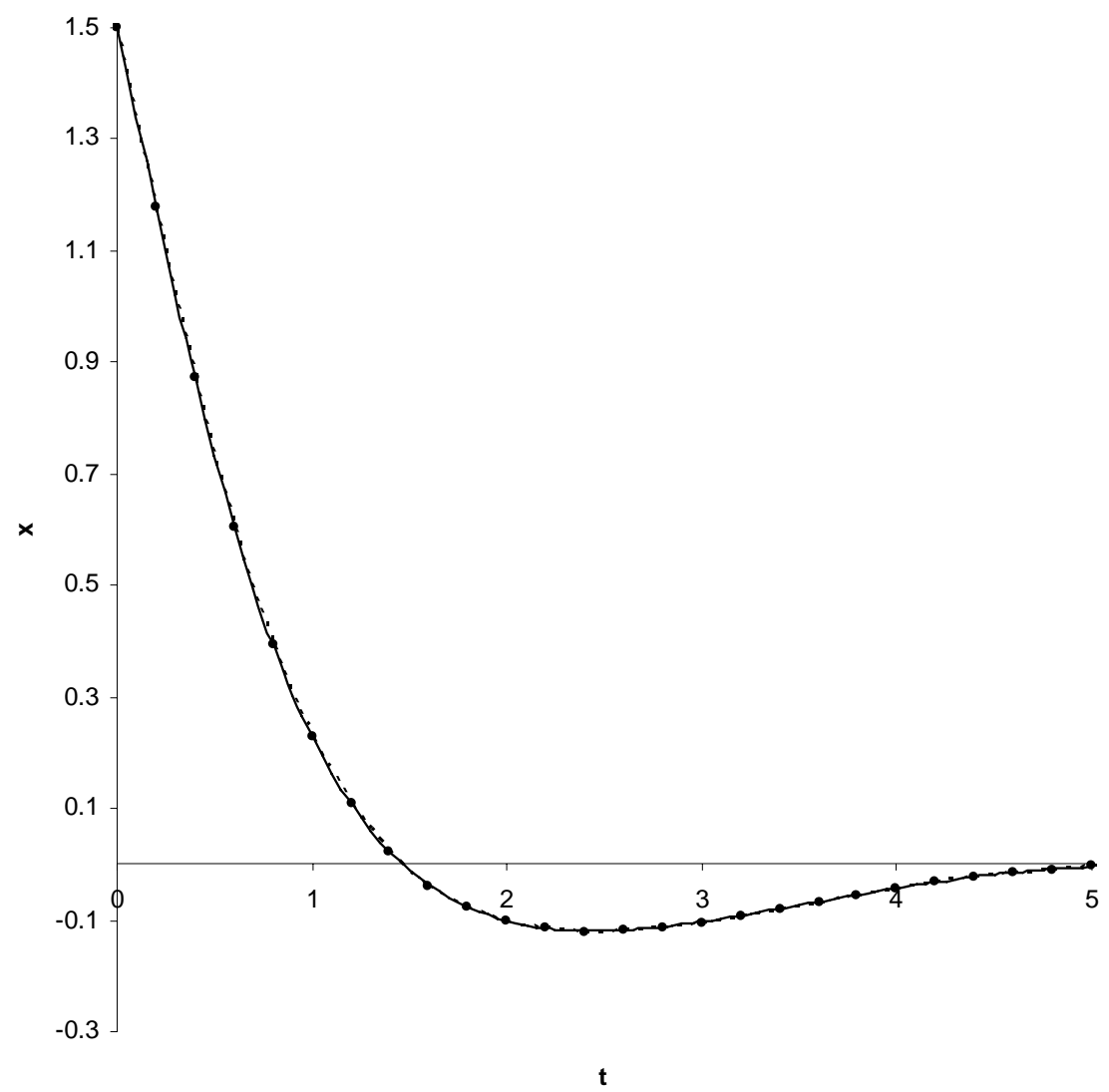

Fig. 2: First approximate solution (denoted by - - -) of Eq. (13), with the initial conditions $[x(0)=$ 1.5000, $\dot{x}(0)-1.55988, \ddot{x}(0)=-1.01827, \dddot{x}(0)=9.2714]$ or $a_{0}=0.5, \varphi_{1}=0, b=1.0, \varphi_{2}$ $=0$, with $\mu_{1}=1.75, \mu_{2}=0.75, \omega_{0}=1.0, h=0.5, \varepsilon=0.1, \omega_{1}=2 \omega_{2}, \omega_{2}=\omega_{0} e^{-h r}, \tau=\varepsilon t$ and $f$ $=x^{3}$.Corresponding numerical solution is denoted by - (solid line).

\section{CONCLUSION}

A unified KBM (Alam 2002, 2003, Bogoliubov et al. 1961, Krylov et al. 1947) method is presented to obtain the analytical approximate solution of a fourth order nonlinear differential system with strong damping and slowly varying coefficients with small nonlinearity. The later form of the solution of Eq. (12) is presented in terms of amplitudes and phases variables. This form is very important in physical problems, since amplitudes and phases characterize the oscillating processes. Moreover the variational equations of amplitudes and phases are important to investigate the stability of a differential system. In general, the variational equations for the amplitudes and phases, namely Eq. (18) is solved numerically. In this case, the perturbation method facilitates the numerical method. The variables $a, \varphi_{1}, b$ and $\varphi_{2}$ change slowly with timet. So it 
requires the numerical calculation of a few numbers of points. On the contrary, a direct attempt to solve Eq. (12) dealing with some harmonic terms in the solution Eq. (22), requires the numerical calculation of a great number of points.

\section{REFERENCES}

Alam, M. S. and M. A. Sattar. 1997. A unified Krylov-Bogoliubov-Mitropolskii method for solving third-order nonlinear systems, Indian Journal of Pure and Applied Mathematics 28: 151-167.

Alam, M. S. 2002. A Unified Krylov-Bogoliubov-Mitropolskii method for solving nth order nonlinear systems, Journal of the Franklin Institute 339: 239-248.

Alam, M. S. 2003. A Unified Krylov-Bogoliubov-Mitropolskii method for solving $n$th order nonlinear systems with slowly varying coefficients, Journal of Sound and Vibration 265: 9871002.

Alam, M. S. and M. A. Sattar. 2004. Asymptotic method for third-order nonlinear systems with slowly varying coefficients, Journal of the Southeast Asian Bulletin of Mathematics 28: 979987.

Arya, J. C. and G. N. Bojadziev. 1980. Damped oscillating systems modeled by hyperbolic differential equations with slowly varying coefficients, Acta Mechanica 35: 215-221.

Arya, J. C. and G. N. Bojadziev. 1981. Time-dependent oscillating systems with damping, slowly varying parameters and delay, Acta Mechanica 41: 109-119.

Akbar, M. Ali, M. S. Alam and M. A. Sattar. 2006. KBM unified method for solving an $n$th order nonlinear differential equation under some special conditions including the internal resonance, International Journal of Nonlinear Mechanics 41: 26-42.

Bogoliubov, N. N. and Yu. A. Mitropolskii. 1961. Asymptotic method in the theory of nonlinear oscillations, Gordan and Breach, New York.

Bojadziev, G. N. and J. Edwards. 1981. On some asymptotic methods for non-oscillatory and oscillatory Processes, Journal of Nonlinear vibration problems 20: 69-79.

Bojadziev, G. N. 1983. Damped nonlinear oscillations modeled by a 3-dimensional differential system, Acta Mechanica 48: 193-201.

Feshchenko, S. F., N. I. Shkil and Nikolenko. 1966. Asymptotic method in the theory of linear differential equation (Russian), Noaukova Dumka, Kiev (English translation, Amer, Elsevier Publishing Co., INC. New York, 1967).

Krylov, N. N. and N. N. Bogoliubov. 1947. Introduction to nonlinear mechanics, Princeton University Press, New Jersey.

Murty, I. S. N., B. L. Deekshatulu and G. Krisna. 1969. On asymptotic method of KrylovBogoliubov method for over damped nonlinear systems, Journal of the Franklin Institute 288: 49-64.

Murty, I. S. N. 1971. A Unified Krylov-Bogoliubov method for solving second-order nonlinear systems, International Journal of Nonlinear Mechanics 6: 45-53.

Popov, I. P. 1956. A generalization of the Bogoliubov asymptotic method in the theory of nonlinear oscillations, Dokl. Akad. Nauk. SSSR, 111: 308-310.

(Received revised manuscript on 10 February, 2010) 